\title{
Dipsogenic Diabetes Insipidus
}

National Cancer Institute

\section{Source}

National Cancer Institute. Dipsogenic Diabetes Insipidus. NCI Thesaurus. Code C129735.

Diabetes insipidus caused by excessive intake of water due to psychological factors or damage to the thirst-regulating mechanism. 